\title{
Produtividade e estabilidade fenotípica de cultivares de milho em três municípios do Estado do Pará(1)
}

\author{
Francisco Ronaldo Sarmanho de Souza ${ }^{(2)}$, Pedro Hélio Estevam Ribeiro(3), \\ Carlos Alberto Costa Veloso(2) e Luiz André Corrêa(4)
}

\begin{abstract}
Resumo - Aárea cultivada com grãos no Estado do Pará está em torno de 553 mil hectares e estima-se que $57 \%$ seja cultivada com milho. A produtividade média do milho é baixa, em virtude dos sistemas de produção adotados, que não incluem o uso de fertilizantes, corretivos e cultivares adaptadas. O objetivo deste trabalho foi avaliar o desempenho produtivo e a estabilidade fenotípica de cultivares de milho em quatro ambientes. Foram avaliadas 37 cultivares comerciais e pré-comerciais de milho em delineamento de látice simples 6x6. As parcelas foram constituídas de duas linhas de $5 \mathrm{~m}$, espaçadas de 0,90 m, com cinco plantas/m após o desbaste. Houve diferença significativa pelo teste $\mathrm{F}$ na maioria das fontes de variação considerada. No grupo das cultivares mais produtivas, a produção esteve entre 5,64 e 6,58 t/ha e no segundo grupo, entre 4,03 e 5,52 t/ha de grãos. A testemunha BR-5102 foi classificada no grupo das mais produtivas, superando mais de $66 \%$ das cultivares avaliadas. As cultivares com menor risco de adoção para recomendação foram a AG 4051, C 333B e 984-N. Os resultados mostram ser possível identificar cultivares com bom desempenho produtivo e boa estabilidade.
\end{abstract}

Termos para indexação: Zea mays, genótipo, desenvolvimento da população, desempenho produtivo.

\section{Yielding and phenotypic stability of corn cultivars in three municipal districts of Pará State, Brazil}

\begin{abstract}
The area cultivated with grains in the State of Pará, Brazil, is around 553 thousand hectares and $57 \%$ of this area is cultivated with corn. Corn average yield is very low due to the production systems adopted which do not include the use of fertilizers, liming and adapted cultivars. The objective of this work was to evaluate the yielding performance and the phenotypic stability of corn cultivars under four environments. Thirty-seven commercial and pre-commercial corn cultivars were used in a simple 6x6 lattice. The plots consisted of two rows of $5 \mathrm{~m}$, spaced $0.90 \mathrm{~m}$ apart with 5 plants $/ \mathrm{m}$ after thinning. There were significant differences by the $\mathrm{F}$ test for most sources of variation taken into account. Within the group of highest yielding cultivars, yield varied from 5.64 to 6.58 ton/ha and within the second group, between 4.03 and 5.52 ton/ha of grains. The variety BR-5102 used as control was classified in the highest-yielding cultivars group, outyielding more than $66 \%$ of the cultivars tested. The cultivars which presented the lowest risk of adoption for recommendation were AG 4051, C 333B and 984-N. The results showed that it is possible to identify cultivars bearing good yielding performance and good stability.
\end{abstract}

Index terms: Zea mays, genotypes, plant establishment, plant performance.

(1) Aceito para publicação em 26 de fevereiro de 2002.

(2) Embrapa-Centro de Pesquisa Agroflorestal da Amazônia Oriental, Caixa Postal 48, CEP 66095-100 Belém, PA.

E-mail: sarmanho@cpatu.embrapa.br, veloso@cpatu.embrapa.br

(3) Embrapa-Centro de Pesquisa Agroflorestal de Roraima, Caixa Postal 133, CEP 69301-970 Boa Vista, RR. E-mail: estevam@cpafrr.embrapa.br

(4) Embrapa-Centro Nacional de Pesquisa de Milho e Sorgo, Caixa Postal 151, CEP 35701-970 Sete Lagoas, MG. E-mail: lacorrea@cnpms.embrapa.br

\section{Introdução}

Os estados da Região Norte, historicamente, não figuram entre os grandes produtores de grãos do País, basicamente em virtude das diretrizes e políticas agrícolas que até pouco tempo colocavam essas culturas em planos secundários. No entanto, sabese que em todos os estados da Região são encontradas amplas áreas de solos com elevado potencial agrícola. Estes solos quando manejados de forma adequada, devidamente corrigidos e fertilizados, 
podem apresentar desempenho produtivo a ponto de concorrer com outros centros produtores, conforme resultados de pesquisa obtidos com grãos no Estado do Pará (El-Husny et al., 1998; Souza et al., 1999c).

Embora a área cultivada com grãos no Estado do Pará ainda não seja tão expressiva (553 mil hectares), espera-se um aumento significativo, tanto em área plantada como em produtividade, uma vez que estão sendo viabilizadas grandes áreas com a cultura da soja e do milho, principalmente nas áreas de cerrado. Estima-se que da área total produzida com grãos, $57 \%$ (314.000 ha) sejam cultivadas com a cultura do milho (Souza et al., 1999). Todavia, a produtividade média desse cereal ainda é muito baixa, dado o baixo índice de adoção de tecnologias modernas, principalmente de fertilizantes, corretivos e de cultivares adaptadas.

Como os programas de melhoramento das grandes empresas de milho não contemplam os estados da Região Norte, as cultivares disponíveis no mercado podem não ser as mais indicadas para cultivo. Neste caso, uma alternativa paralela a um programa de melhoramento, que pode trazer resultados satisfatórios, seria a introdução e avaliação de cultivares pré-comerciais e comerciais em vários municípios representativos do estado. As condições edafoclimáticas encontradas no Estado do Pará, de maneira geral, não apresentam limitações ao desenvolvimento da cultura do milho, mas podem ser diferentes, de forma significativa, entre os municípios. Por isso, seria interessante, durante as avaliações de cultivares, verificar os efeitos da interação genótipos x ambientes. Esses efeitos da interação, além de dificultar os trabalhos dos melhoristas na seleção de genótipos efetivamente superiores, também interferem na recomendação de cultivares e isto tem-se constatado com frequiência na literatura (Ramalho et al., 1990; Gonçalves et al., 1999; Ribeiro et al., 1999).

O milho, por ser um cereal cultivado de norte a sul do Brasil, é submetido a uma grande diversidade de condições ambientais, e com isto, é de se esperar que haja o efeito da interação de genótipos com ambientes, que é percebida quando há comportamento diferencial das cultivares nas diferentes condições ambientais a que são submetidas. As alternativas mais comumente utilizadas pelos melhoristas são a obtenção de cultivares específicas para cada am- biente, estratificação de ambientes e identificação de cultivares com maior estabilidade fenotípica (Vencovsky \& Barriga, 1992; Ramalho et al., 1993).

No entanto, no caso de culturas anuais, como o milho, os estudos têm-se concentrado na estabilidade das cultivares. Neste sentido, existem inúmeros métodos que possibilitam identificar os materiais mais estáveis. Alguns desses métodos são relatados por Cruz \& Regazzi (1994). Um método muito prático para o caso de recomendação de cultivares foi apresentado por Annicchiarico (1992), e já tem sido utilizado no estudo de estabilidade de cultivares de milho no Brasil (Ribeiro, 1998; Gonçalves et al., 1999). Este método apresenta um índice de confiança (Index of reliability) que propicia recomendar as cultivares aos agricultores, considerando o risco de apresentarem desempenho abaixo de um dado padrão, que pode ser, por exemplo, a média geral. Quanto maior for o índice de confiança de uma dada cultivar, menor será sua probabilidade de insucesso.

O objetivo deste trabalho foi avaliar o desempenho produtivo e a estabilidade fenotípica de cultivares de milho em quatro ambientes no Estado do Pará.

\section{Material e Métodos}

Foram avaliadas 37 cultivares comerciais e pré-comerciais de várias instituições de pesquisa do País, utilizandose como testemunha a variedade BR-5102 melhorada na região. $\mathrm{O}$ delineamento experimental utilizado foi o de látice simples 6x6, com a testemunha local aleatorizada em cada repetição. A área útil das parcelas foi constituída de duas linhas de $5 \mathrm{~m}$ com espaçamento de $0,90 \mathrm{~m}$ com cinco plantas por metro após o desbaste. Os experimentos foram conduzidos nos municípios de Paragominas, Alenquer e Redenção; em Paragominas, foram conduzidos dois experimentos no mesmo ano. Na Tabela 1 são apresentadas as características dos ambientes onde as avaliações foram realizadas.

A adubação utilizada em todos os locais foi o equivalente a $400 \mathrm{~kg} / \mathrm{ha}$ de $\mathrm{N}, \mathrm{P}$, e $\mathrm{K}$ na fórmula NPK 10-28$20+0,4 \%$ de $\mathrm{Zn}$, aplicados por ocasião do plantio, mais $100 \mathrm{~kg} /$ ha de uréia em cobertura, aplicados a lanço aos 30 dias após o plantio. As demais práticas culturais foram realizadas conforme recomendado para a cultura na região (Souza et al., 1999c). Foram avaliadas as variáveis: floração masculina (FLO), altura da planta (ALP), altura da espiga (ALE), número de espigas (NES) e peso de grãos (PGR). O caráter peso de grãos foi corrigido para umidade de $13 \%$ e para estande ideal de 50 plantas/parcela, utilizando-se o procedimento apresentado por Vencovsky \& Barriga 
(1992). Primeiramente, fizeram-se as análises individuais seguindo os procedimentos para o delineamento em látice, sem a inclusão da cultivar testemunha. Após ter-se verificado que a eficiência do látice (96\% a 102\%) em relação ao delineamento de bloco foi relativamente baixa, optou-se por realizar as análises individuais em blocos ao acaso, incluindo-se a testemunha. Com as médias das 37 cultivares em cada ambiente, e após ter-se verificado que não houve problemas de homogeneidade de variância residual, realizaram-se as análises conjuntas e de estabilidade, pelo método proposto por Annicchiarico (1992). Para discriminação das médias entre as cultivares, utilizou-se o teste de Scott \& Knott (1974), por ser um teste de análise em cluster que apresenta a vantagem de não sobrepor as classes, onde cada grupamento de médias recebe apenas uma mesma letra, facilitando a visualização e interpretação dos resultados.

Com as médias de cada ambiente fez-se a análise de estabilidade, pelo método proposto por Annicchiarico (1992). As análises foram realizadas utilizando-se o Software Sisvar e Estabilidade (Ferreira \& Zambalde, 1997).

\section{Resultados e Discussão}

Houve significância, a 5\% pelo teste F, na maioria das fontes de variação considerada, à exceção de repetições dentro de ambiente, quanto às variáveis floração masculina e altura de espigas, quanto à interação cultivares $\mathrm{x}$ ambientes, e quanto à altura da planta (Tabela 2). A significância verificada nas fontes de variação cultivar, ambientes e interação cultivares $\mathrm{x}$ ambientes, mostra que houve diferença no comportamento das cultivares, na manifestação ambiental, e que, de um local para outro, as cultivares mostraram manifestação fenotípica inconsistente perante as variações ambientais. O efeito desta interação tem sido estudado em todas as regiões do País, tanto para os diversos tipos de cultivares existentes no mercado, como para vários tipos de famílias obtidos na cultura do milho (Carvalho et al., 1992; Arias, 1996; Ribeiro et al., 1999).

As estimativas dos coeficientes de variação experimental variaram de $2,91 \%$ a $19,84 \%$ quanto a floração e rendimento de grãos, respectivamente. Tratando-se de experimentos de cultivares de milho, estas estimativas indicam que não houve maiores problemas durante a sua execução. Embora a média geral de produtividade de grãos (5,53 t/ha) dos experimentos esteja um pouco abaixo das médias obtidas em outros centros do País, tem-se que esse valor supera em mais de $100 \%$ a média do Estado do Pará, que está em torno de 1,4 t/ha (Souza et al., 1999a, 1999b, 1999c).

Tabela 1. Características edafoclimáticas dos municípios de Alenquer, Paragominas e Redenção no Estado do Pará, 2000 .

\begin{tabular}{|c|c|c|c|c|c|}
\hline Local & $\begin{array}{l}\text { Precipitação } \\
(\mathrm{mm})\end{array}$ & $\begin{array}{l}\text { Altitude } \\
(\mathrm{m})\end{array}$ & Latitude & Longitude & Solo \\
\hline Alenquer & 1.900 & 52 & $1^{\circ} 56^{\prime} 45^{\prime \prime}$ & $54^{\circ} 44^{\prime} 38^{\prime \prime}$ & Vertissolo Ebânico órtico típico, textura argilosa \\
\hline Paragominas & 1.800 & 90 & $2^{\circ} 59^{\prime} 29^{\prime \prime}$ & $47^{\prime} 21^{\prime} 6^{\prime \prime}$ & $\begin{array}{l}\text { Latossolo Amarelo distrófico típico, textura muito } \\
\text { argilosa }\end{array}$ \\
\hline Redenção & 1.700 & 150 & $8^{\circ} 2^{\prime} 16^{\prime \prime}$ & $50^{\circ} 2^{\prime} 48^{\prime \prime}$ & $\begin{array}{l}\text { Latossolo Vermelho-Amarelo distrófico } \\
\text { cascalhento, textura argilosa }\end{array}$ \\
\hline
\end{tabular}

Tabela 2. Análise de variância para as variáveis peso de grãos (PGR) em t/ha, dias para floração masculina (FLO), altura da planta (ALP), altura da espiga (ALE) e número de espigas (NES), de 37 cultivares de milho, em quatro ambientes do Estado do Pará, 2000.

\begin{tabular}{|c|c|c|c|c|c|c|}
\hline Fontes de variação & GL & PGR & FLO & ALP & ALE & NES \\
\hline Cultivar (C) & 36 & $2,04 * *$ & $14,06 * *$ & $1.253,50 * *$ & $537,57 * *$ & $47,29 * *$ \\
\hline Ambiente (A) & 3 & $115,30 * *$ & $1.073,92 * *$ & $568,40 * *$ & $7.409,39 * *$ & $513,26 * *$ \\
\hline $\mathrm{C} \times \mathrm{A}$ & 108 & $1,32 * *$ & $6,80 * *$ & $249,65^{\mathrm{ns}}$ & $185,76^{*}$ & $35,16 * *$ \\
\hline Repetição & 4 & $4,92 * *$ & $0,34^{\mathrm{ns}}$ & $954,18 * *$ & $123,15^{\mathrm{ns}}$ & $66,55^{* *}$ \\
\hline Erro & 144 & 0,95 & 2,18 & 217,40 & 138,30 & 22,35 \\
\hline $\mathrm{CV}(\%)$ & & 19,84 & 2,91 & 7,13 & 10,93 & 10,60 \\
\hline Média & & 5,53 & 50,70 & 206,62 & 107,62 & 44,63 \\
\hline
\end{tabular}

nsNão-significativo. * e ${ }^{* *}$ Significativo a $5 \%$ e a $1 \%$ de probabilidade, respectivamente, pelo teste $\mathrm{F}$. 
Pelo teste de Scott \& Knott (1974) a 5\% de probabilidade, e pelo índice de confiança de Annicchiarico (1992), verificou-se que as cultivares foram classificadas em apenas dois grupos com relação aos caracteres altura da espiga, número de espigas e peso de grãos; já com relação à floração e altura das plantas, os materiais foram classificados em quatro e três grupos, respectivamente (Tabela 3). Dentro do grupo das cultivares mais produtivas, a produção esteve entre 5,64 e 6,58 t/ha, e, no segundo grupo, entre 4,03 e 5,52 t/ha de grãos. O que chama a atenção no caso de peso de grãos é o desempenho da cultivartestemunha BR-5102, que foi classificada no grupo das mais produtivas, superando em mais de $66 \%$ as

Tabela 3. Floração masculina (FLO), altura da planta (ALP), altura da espiga (ALE), número de espigas (NES) e peso de grãos (PGR), com a classificação obtida pelo teste de Scott \& Knott (1974) e índice de confiança (Ii\%) de Annicchiarico (1992) de 37 cultivares de milho avaliadas em quatro ambientes do Estado do Pará, 2000 ${ }^{(1)}$.

\begin{tabular}{|c|c|c|c|c|c|c|c|c|c|c|}
\hline Cultivar & $\begin{array}{l}\text { FLO } \\
\text { (dias) }\end{array}$ & $\begin{array}{c}\mathrm{Ii} \\
(\%)\end{array}$ & $\begin{array}{l}\text { ALP } \\
(\mathrm{cm})\end{array}$ & $\begin{array}{c}\mathrm{Ii} \\
(\%)\end{array}$ & $\begin{array}{l}\text { ALE } \\
(\mathrm{cm})\end{array}$ & $\begin{array}{c}\mathrm{Ii} \\
(\%)\end{array}$ & NES & $\begin{array}{c}\mathrm{Ii} \\
(\%)\end{array}$ & PGR & $\begin{array}{c}\mathrm{Ii} \\
(\%)\end{array}$ \\
\hline AG 4051 & $50,00 \mathrm{~d}$ & 100,57 & $228,38 \mathrm{a}$ & 113,82 & $120,25 \mathrm{a}$ & 114,41 & $45,75 \mathrm{a}$ & 102,80 & $6,58 \mathrm{a}$ & 123,50 \\
\hline $984-\mathrm{N}$ & $50,88 \mathrm{c}$ & 99,86 & $202,63 c$ & 95,49 & $102,13 b$ & 92,65 & $48,13 \mathrm{a}$ & 113,89 & $6,58 \mathrm{a}$ & 119,21 \\
\hline C 333B & $51,75 \mathrm{~b}$ & 101,27 & $201,00 \mathrm{c}$ & 99,43 & $106,25 b$ & 100,08 & $45,13 \mathrm{a}$ & 98,93 & $6,53 a$ & 121,00 \\
\hline CX 9805 & $49,63 d$ & 99,17 & $193,75 \mathrm{c}$ & 95,57 & $101,63 b$ & 96,90 & $44,75 \mathrm{a}$ & 100,18 & $6,14 a$ & 112,97 \\
\hline X $1286 \mathrm{~K}$ & $48,88 \mathrm{~d}$ & 96,92 & $193,75 \mathrm{c}$ & 95,13 & $100,38 \mathrm{~b}$ & 92,67 & $48,13 \mathrm{a}$ & 106,48 & $6,10 \mathrm{a}$ & 111,04 \\
\hline HT 970556 & $51,50 \mathrm{c}$ & 101,97 & $193,25 c$ & 90,90 & $107,13 b$ & 98,08 & $48,50 \mathrm{a}$ & 109,47 & $6,08 \mathrm{a}$ & 106,39 \\
\hline XB 7012 & $52,88 \mathrm{a}$ & 102,97 & $211,50 \mathrm{~b}$ & 106,52 & $107,50 \mathrm{~b}$ & 103,48 & $44,75 \mathrm{a}$ & 96,68 & $5,97 \mathrm{a}$ & 111,53 \\
\hline P 3027 & $50,88 \mathrm{c}$ & 97,04 & $195,00 \mathrm{c}$ & 96,75 & $100,13 b$ & 94,53 & $44,88 \mathrm{a}$ & 98,89 & $5,84 a$ & 107,09 \\
\hline Z 85E03 & $48,25 \mathrm{~d}$ & 95,53 & $185,13 \mathrm{c}$ & 89,47 & $91,00 \mathrm{~b}$ & 86,84 & $44,75 \mathrm{a}$ & 99,85 & $5,84 a$ & 108,94 \\
\hline MTL 9826 & $53,88 \mathrm{a}$ & 106,48 & $210,13 b$ & 107,41 & $117,38 \mathrm{a}$ & 108,28 & $43,50 b$ & 95,22 & $5,76 a$ & 101,68 \\
\hline IAC $0410 \mathrm{E}$ & $48,38 \mathrm{~d}$ & 94,12 & $206,13 c$ & 98,65 & $108,75 b$ & 101,44 & $45,63 \mathrm{a}$ & 103,63 & $5,73 \mathrm{a}$ & 105,35 \\
\hline BR-5102 & $52,25 \mathrm{~b}$ & 102,00 & $226,13 a$ & 108,00 & $118,25 \mathrm{a}$ & 110,00 & $47,13 \mathrm{a}$ & 106,00 & $5,70 \mathrm{a}$ & 107,51 \\
\hline MTL 9744 & $51,88 \mathrm{~b}$ & 100,54 & $184,25 \mathrm{c}$ & 92,18 & $94,25 b$ & 88,00 & $44,38 \mathrm{a}$ & 99,80 & $5,64 \mathrm{a}$ & 97,53 \\
\hline EXP 2 & $49,88 \mathrm{~d}$ & 101,97 & $208,13 b$ & 97,58 & $99,13 b$ & 94,38 & $45,13 \mathrm{a}$ & 98,91 & $5,52 b$ & 101,03 \\
\hline 97HT128QPM & $49,25 \mathrm{~d}$ & 97,04 & $209,63 b$ & 97,52 & $106,63 b$ & 96,56 & $47,38 \mathrm{a}$ & 106,48 & $5,51 \mathrm{~b}$ & 99,68 \\
\hline P 3021 & $51,13 \mathrm{c}$ & 102,66 & $192,75 \mathrm{c}$ & 93,09 & $100,63 b$ & 93,79 & $45,50 \mathrm{a}$ & 107,31 & $5,50 \mathrm{~b}$ & 98,22 \\
\hline Z 85E50 & $49,25 \mathrm{~d}$ & 99,04 & $196,63 c$ & 93,75 & $103,38 b$ & 94,30 & $46,63 \mathrm{a}$ & 105,99 & $5,50 \mathrm{~b}$ & 101,30 \\
\hline HD 9555 & $51,25 \mathrm{~d}$ & 97,05 & $206,13 c$ & 95,75 & $105,50 \mathrm{~b}$ & 99,48 & $42,38 \mathrm{a}$ & 102,02 & $5,46 \mathrm{~b}$ & 99,31 \\
\hline BRS 3060 & $50,38 \mathrm{c}$ & 99,87 & $198,75 \mathrm{c}$ & 97,63 & $107,63 b$ & 97,14 & $45,00 \mathrm{~b}$ & 86,31 & $5,46 \mathrm{~b}$ & 92,80 \\
\hline AL CG5 & $50,50 \mathrm{c}$ & 100,57 & $220,88 \mathrm{a}$ & 106,91 & $118,13 \mathrm{a}$ & 110,35 & $39,50 \mathrm{~b}$ & 85,92 & $5,45 b$ & 99,73 \\
\hline CX 9806 & $50,63 \mathrm{c}$ & 98,47 & $204,00 \mathrm{c}$ & 103,42 & $98,00 \mathrm{~b}$ & 92,90 & $46,63 \mathrm{a}$ & 102,70 & $5,42 b$ & 99,33 \\
\hline 96НT91QPM & $50,75 \mathrm{c}$ & 100,57 & $225,13 a$ & 106,10 & $121,25 \mathrm{a}$ & 114,61 & $45,00 \mathrm{a}$ & 102,00 & $5,38 b$ & 96,51 \\
\hline BRS 2110 & $49,25 \mathrm{~d}$ & 97,64 & $204,13 c$ & 97,94 & $103,63 b$ & 95,24 & $43,38 b$ & 102,92 & $5,34 b$ & 97,35 \\
\hline 97 HT129QPM & $50,88 \mathrm{c}$ & 100,58 & $233,50 \mathrm{a}$ & 114,19 & $115,75 \mathrm{a}$ & 107,00 & $41,25 b$ & 90,58 & $5,29 \mathrm{~b}$ & 100,41 \\
\hline P $30 F 80$ & $50,75 \mathrm{c}$ & 98,47 & $200,50 \mathrm{c}$ & 96,61 & $108,00 \mathrm{~b}$ & 101,59 & $47,13 a$ & 109,03 & $5,24 b$ & 95,40 \\
\hline HT 111301 & $51,00 \mathrm{c}$ & 99,17 & $215,88 b$ & 106,18 & $111,38 \mathrm{a}$ & 106,43 & $47,00 \mathrm{a}$ & 109,53 & $5,21 b$ & 96,08 \\
\hline $985-\mathrm{N}$ & $49,25 \mathrm{~d}$ & 99,17 & $205,13 c$ & 101,39 & $113,75 \mathrm{a}$ & 104,82 & $46,38 \mathrm{a}$ & 103,34 & $5,18 b$ & 98,83 \\
\hline Z 85E02 & $48,88 \mathrm{~d}$ & 98,35 & $191,13 \mathrm{c}$ & 90,75 & $95,75 b$ & 91,15 & $38,63 b$ & 77,45 & $5,13 b$ & 97,47 \\
\hline BRS 2114 & $51,75 \mathrm{~b}$ & 100,55 & $195,25 \mathrm{c}$ & 100,00 & $100,00 \mathrm{~b}$ & 94,15 & $45,75 \mathrm{a}$ & 108,21 & $5,10 \mathrm{~b}$ & 86,95 \\
\hline AGROMEN 1E1 & $49,63 \mathrm{~d}$ & 99,04 & $196,63 \mathrm{c}$ & 93,85 & $99,13 b$ & 93,24 & $45,00 \mathrm{a}$ & 100,62 & $5,08 \mathrm{~b}$ & 90,33 \\
\hline CO-9621 & $53,00 \mathrm{a}$ & 104,39 & $227,75 a$ & 107,74 & $119,75 a$ & 106,24 & $45,13 \mathrm{a}$ & 102,87 & $4,98 \mathrm{~b}$ & 99,88 \\
\hline AL MANDURI/XV & $51,25 \mathrm{c}$ & 99,14 & $217,50 \mathrm{~b}$ & 105,89 & $119,13 \mathrm{a}$ & 106,11 & $44,63 a$ & 103,37 & $4,89 \mathrm{~b}$ & 91,38 \\
\hline $\mathrm{R} \& \mathrm{G} 02 \mathrm{E}$ & $51,25 \mathrm{c}$ & 101,27 & $219,75 a$ & 105,06 & $118,75 \mathrm{a}$ & 110,53 & $42,63 b$ & 102,76 & $4,86 \mathrm{~b}$ & 91,35 \\
\hline CATI AL30/IX & $50,88 \mathrm{c}$ & 100,57 & $213,38 b$ & 103,39 & $106,75 b$ & 100,33 & $39,88 b$ & 89,12 & $4,81 \mathrm{~b}$ & 86,31 \\
\hline AL 25/XV & $52,00 \mathrm{~b}$ & 102,66 & $214,00 \mathrm{~b}$ & 104,59 & $113,25 \mathrm{a}$ & 107,14 & $41,63 b$ & 88,42 & $4,57 b$ & 85,20 \\
\hline AL 34/XV & $51,75 \mathrm{~b}$ & 101,97 & $209,75 b$ & 99,01 & $114,25 \mathrm{a}$ & 103,21 & $42,00 \mathrm{~b}$ & 93,73 & $4,56 \mathrm{~b}$ & 87,85 \\
\hline IAC PARIQUERA & $52,25 \mathrm{~b}$ & 103,36 & $209,25 b$ & 100,29 & $108,00 \mathrm{~b}$ & 101,94 & $42,00 \mathrm{~b}$ & 94,58 & $4,03 \mathrm{~b}$ & 73,55 \\
\hline Máximo & 53,88 & 106,48 & 233,50 & 114,19 & 121,25 & 114,61 & 48,50 & 113,89 & 6,58 & 123,50 \\
\hline Mínimo & 48,25 & 94,12 & 184,25 & 89,47 & 91,00 & 86,84 & 38,63 & 77,45 & 4,03 & 73,55 \\
\hline
\end{tabular}

${ }^{(1)}$ Médias seguidas de mesmas letras não diferem entre si pelo teste de Scott \& Knott, a $5 \%$ de probabilidade. 
cultivares testadas. Isto mostra que o fato de uma cultivar ser híbrida não a torna necessariamente mais produtiva.

A superioridade de uma cultivar em relação a cultivares híbridas já foi verificada em outras ocasiões por Gomes (1990) e Ribeiro et al. (1999), uma vez que o desempenho de uma cultivar pode se dar em função da homeostase individual, que predomina no caso dos híbridos simples ou homeostase populacional, que ocorre em cultivares de polinização livre (Allard \& Bradshaw, 1964). A cultivar-testemunha já vem sendo melhorada na própria região, o que, de certa forma, atenua os efeitos da interação genótipos $\mathrm{x}$ ambientes. Por outro lado, considerando-se o índice proposto por Annicchiarico (1992), verifica-se que cultivares desenvolvidas em outras regiões do País apresentaram maior estabilidade, e portanto, menor risco de adoção pelos produtores; o híbrido triplo AG 4051 com $\mathrm{Ii}=123 \%$, seguido pelas cultivares C 333B e 984-N com Ii=121 e Ii=119\%, respectivamente, atingiram os melhores índices de confiança.

Normalmente, quando cultivares desenvolvidas por outras regiões do País, como Sul e Sudeste e até mesmo Centro-Oeste, são introduzidas em outra região, sofrem uma redução no ciclo vegetativo, e, dependendo da região, esta redução é bem significativa (Numberg, 2000; Ribeiro et al., 2000b). Ribeiro et al. (2000a) comentam que em Roraima, os materiais com ciclo mais longo sofrem maior redução que os precoces, quando introduzidos. Talvez isto explique a pouca diferença entre as médias máxima e mínima obtidas quanto a esta característica. Quanto às demais variáveis, não se observou essa redução.

Alguns autores conceituam adaptabilidade como sendo a capacidade da cultivar de aproveitar vantajosamente o estímulo ambiental quanto à maior produtividade, enquanto estabilidade estaria relacionada à previsibilidade do comportamento em função da qualidade do estímulo ambiental (Mariotti et al., 1976; Verma et al., 1978). Embora essas duas características nem sempre estejam associadas em um mesmo genótipo, as cultivares com maior rendimento médio de grãos foram as dotadas de maior estabilidade ou índice de confiança Ii $(\%)$, cuja correlação entre esses dois parâmetros foi da ordem de $95 \%$ (Tabela 4).
Tabela 4. Correlação fenotípica entre as variáveis floração masculina (FLO) em dias, altura da planta (ALP) em cm, altura da espiga (ALE) em cm, número de espigas (NES) e peso de grãos (PGR) em t/ha, avaliadas duas a duas e entre médias das características e as respectivas estimativas do índice de confiança (Ii\%) de Annichiarico, em 37 cultivares de milho avaliadas em quatro ambientes do Estado do Pará, 2000.

\begin{tabular}{lccccc}
\hline Variável & ALP & ALE & NES & PGR & R Ii $(\%)$ \\
\hline FLO & $30,90^{*}$ & $37,00^{*}$ & $-10,20^{\mathrm{ns}}$ & $-8,30^{\mathrm{ns}}$ & $80,40^{* *}$ \\
ALP & & $87,10^{* *}$ & $-22,00^{\mathrm{ns}}$ & $-21,10^{\mathrm{ns}}$ & $91,80^{* *}$ \\
ALE & & & $-13,10^{\mathrm{ns}}$ & $-20,30^{\mathrm{ns}}$ & $96,10^{* *}$ \\
NES & & & & $49,50^{* *}$ & $83,10^{* *}$ \\
PGR & & & & & $95,20^{* *}$ \\
\hline
\end{tabular}

ns Não-significativo. * e **Significativo a $5 \%$ e a $1 \%$ de probabilidade, respectivamente, pelo teste $\mathrm{t}$.

\section{Conclusões}

1. A etapa de introdução e avaliação de cultivares deve ser realizada para auxiliar na escolha de cultivares mais produtivas e de maior estabilidade para uma dada região.

2. É possível identificar cultivares, geradas em outras regiões do País, com bom desempenho produtivo no Estado do Pará.

3. Considerando-se a média de produtividade e o índice de estabilidade, os melhores materiais são as cultivares AG 4051, C 333B e 984-N.

\section{Referências}

ALLARD, R. W.; BRADSHAW, A. D. Implications of genotype-environmental interaction in applied plant breeding. Crop Science, Madison, v. 4, p. 503-508, 1964.

ANNICCHIARICO, P. Cultivar adaptation and recommendation from alfalfa trials in Northern Italy. Journal of Genetics \& Breeding, Rome, v. 46, n. 1, p. 269-278, Mar. 1992.

ARIAS, E. R. A. Adaptabilidade e estabilidade das cultivares de milho avaliadas no Estado de Mato Grosso do Sul e avanço genético obtido no período de 1986/87 a 1993/94. 1996. $118 \mathrm{f}$. Tese (Doutorado em Fitotecnia) - Universidade Federal de Lavras, Lavras.

CARVALHO, H. W. L. de; MAGNAVACA, R.; LEAL, M. L. S. Estabilidade da produção de cultivares de milho no Estado de Sergipe. Pesquisa Agropecuária Brasileira, Brasília, v. 27, n. 7, p. 1073-1082, jul. 1992. 
CRUZ, C. D.; REGAZZI, A. J. Modelos biométricos aplicados ao melhoramento genético. Viçosa, MG: Imprensa Universitária, 1994. 390 p.

EL-HUSNY, J. C.; ANDRADE, E. B.; MEYER, M. C. Avaliação de cultivares de soja em Paragominas-Pará. Belém: Embrapa-CPATU, 1998. 4 p. (Pesquisa em Andamento, 196).

FERREIRA, D. F.; ZAMBALDE, A. L. Simplificação de algumas técnicas especiais da experimentação agropecuária no MAPGEN e softwares correlatos. In: CONGRESSO DA SOCIEDADE BRASILEIRA DE INFORMÁTICA, 1997, Belo Horizonte. Anais... Belo Horizonte: Sociedade Brasileira de Informática, 1997. p. 285-291.

GOMES, L. S. Interação genótipo $\mathbf{x}$ época de plantio em milho (Zea mays L.) em dois locais do oeste do Paraná. 1990. 148 f. Dissertação (Mestrado) - Escola Superior de Agricultura Luiz de Queiroz, Piracicaba.

GONÇALVES, F. M. A.; CARVALHO, S. P.; RAMALHO, M. A. P.; CORRÊA, L. A. Importância das interações cultivares $\mathrm{x}$ anos na avaliação de milho safrinha. Pesquisa Agropecuária Brasileira, Brasília, v. 34, n. 7, p. 1175-1181, jul. 1999.

MARIOTTI, J. A.; OYARZABAL, E. S.; OSA, J. M.; BULATCIO, A. N. R.; ALMADA, G. H. Análisis de estabilidad y adaptabilidad de genotipos de caña de azúcar I: interacciones dentro de una localidad experimental. Revista Agronómica del Noroeste Argentino, Tucumán, v. 13 , n. 14 , p. 105-127, ene. 1976.

NUMBERG, P. L. Desempenho de híbridos simples com testadores de linhagens de milho em 'top cross'. 2000. 69 f. Dissertação (Mestrado) - Universidade Federal de Lavras, Lavras.

RAMALHO, M. A. P.; SANTOS, J. B.; PINTO, C. A. Genética na agropecuária. São Paulo: Globo, 1990. 359 p.

RAMALHO, M. A. P.; SANTOS, J. B. dos; ZIMMERMANN, M. J. O. Genética quantitativa em plantas autógamas: aplicações ao melhoramento do feijoeiro. Goiânia: UFG, 1993. 271 p.

RIBEIRO, P. H. E. Adaptabilidade e estabilidade de milho em diferentes épocas de semeadura, níveis de adubação e locais do Estado de Minas Gerais. 1998. 126 f.
Tese (Doutorado) - Universidade Federal de Lavras, Lavras.

RIBEIRO, P. H. E.; NUMBERG, P. L.; RAMALHO, M. A. P.; SOUZA, J. C. Avaliação de híbridos simples em cruzamentos 'top cross' com linhagens de milho em Boa Vista, Roraima. Boa Vista: Embrapa-CPAF-RR, 2000a. 27 p. (Boletim de Pesquisa, 1).

RIBEIRO, P. H. E.; RAMALHO, M. A. P.; SOUZA, J. C. Desempenho produtivo de populações de milho em Boa Vista, Roraima. Boa Vista: Embrapa-CPAF-RR, 2000b. 27 p. (Boletim de Pesquisa, 2).

RIBEIRO, P. H. E.; SANTOS, M. X.; RAMALHO, M. A. P. Interação cultivares de milho x épocas de semeadura em diferentes ambientes do Estado de Minas Gerais. Revista Ceres, Viçosa, MG, v. 46, n. 267, p. 531-542, 1999.

SCOTT, A. J.; KNOTT, M. A. Cluster analysis method for grouping means in the analysis of variance. Biometrics, Washington, v. 30, n. 3, p. 507-512, Sept. 1974.

SOUZA, F. R. S. de; VELOSO, C. A. C.; POLTRONIERI, L. S. P.; ARAÚJO, S. M. B. Avaliação do comportamento de híbridos comerciais de milho nas regiões nordeste e sudeste do Estado do Pará. Belém: Embrapa-CPATU, 1999a. 4 p. (Pesquisa em Andamento, 214).

SOUZA, F. R. S.; VELOSO, C. A. C.; GAMA, E. E. G.; CORRÊA, L. A.; RIBEIRO, S. I. Comportamento de 36 híbridos comerciais de milho na Região Oeste do Pará. In: REUNIÓN LATINOAMERICANA DEL MAÍZ, 28., 1999, Sete Lagoas. Memórias... Sete Lagoas: EmbrapaCNPMS/Centro Internacional de Mejoramiento de Maíz y Trigo, 1999b. p. 583-586.

SOUZA, F. R. S. de; VELOSO, C. A. C.; POLTRONIERI, L. S.; ARAÚJO, S. M. B. de. Recomendações básicas para o cultivo do milho no Estado do Pará. Belém: Embrapa-CPATU, 1999c. 20 p. (Circular Técnica, 2).

VENCOVSKY, R.; BARRIGA, P. Genética biométrica no fitomelhoramento. Ribeirão Preto: Sociedade Brasileira de Genética, 1992. 496 p.

VERMA, M. M.; CHAHAL, G. S.; MURTY, B. R. Limitation of conventional regression analysis: a proposed modification. Theoretical and Applied Genetics, New York, v. 53, n. 1, p. 89-91, Jan. 1978. 